\title{
A Repeated Median Filtering Method for Denoising Mammogram Images
}

\author{
Hussain AlSalman \\ Department of Computer Science \\ College of Computer and Information Sciences \\ King Saud University, Riyadh, Saudi Arabia
}

\begin{abstract}
In the medical field, mammogram analysis is one of the most important breast cancer detection procedures and early diagnosis. During the image acquisition process of mammograms, the acquired images may be contained some noises due to the change of illumination and sensor error. Hence, it is necessary to remove these noises without affecting the edges and fine details, achieving an effective diagnosis of beast images. In this work, a repeated median filtering method is proposed for denoising digital mammogram images. A number of experiments are conducted on a dataset of different mammogram images to evaluate the proposed method using a set of image quality metrics. Experimental results are reported by computing the image quality metrics between the original clean images and denoised images that are corrupted by different levels of simulated speckle noise as well as salt and paper noise. Evaluation quality metrics showed that the repeated median filter method achieves a higher result than the related traditional median filter method.
\end{abstract}

Keywords-Mammogram images; image denoising; median filter; repeated median filtering; speckle noise; salt and paper noise

\section{INTRODUCTION}

Nowadays, image processing methods have been applied for diagnosis in several medical applications, such as liver image analysis [1, 2], brain tumor classification [3, 4], breast image enhancement, and cancer diagnosis [5-7], and so on. Image denoising process is used to eliminate noises from noisy images and improve their quality. However, it faces difficulty to distinguish between noises and other important images' components such as edges and textures due to they have approximately the same high frequencies, which might lead to lose some details of the images [8]. Therefore, image denoising without losing significant information from a noisy image is still a vital problem in the image processing field [8]. In recent years, great achievement has been accomplished in the field of image denoising [9-12].

In medical imaging systems and applications, image denoising plays an important role as a pre-processing step to enhance the quality of digital images and improving the process of medical diagnosis [13]. Even though the medical image denoising process has been studied in many research types for a long time, it is still a challenging issue and an open task. One of the key reasons for this is that the medical image denoising is an inverse problem from a mathematical perspective, and its solution is not unique and not flexible.
The rest of the paper is organized as follows: Section II presents a literature review about the methods used for medical and breast image denoising. Section III describes the applied research methods. Section IV presents the experimental results and discussion. Finally, Section V concludes and discusses the research work.

\section{LITERATURE REVIEW}

Currently, there are many approaches to image denoising for medical imaging systems. Some of the common approaches are median filtering, Wiener filtering, morphological filtering, wavelet-based filtering, and curvelet transform, among other significant approaches. The median filter $[14,15]$ is a statistical approach for noise reduction in images with blurred edges. Wiener filter is another statistical approach that calculates unknown signals using a related known signal as input [16]. Morphological filtering is a local non-linear transformation of geometric features; its fundamental operations are closing, opening, erosion, and dilation. It has been applied in different areas, especially image denoising [17]. Wavelet-based filtering has also been used for the d-noising of images of all kinds, specifically for medical image systems. It is a mathematical calculation that is able to perceive local features of the image.

Additionally, it is used to decompose 2D signals into diverse resolution levels [18]. For a functional performance of image denoising, an adaptive procedure for image discontinuities are applied. Accordingly, a multi-resolution approach is adapted. Here, the curvelet transform can be used to improve image resolution [19]. Breast cancer is currently the most common type of cancer with the highest mortality cause among women in the world ${ }^{1}$. The number of deaths from breast cancer has doubled in 22 years, affecting both industrialized and less developed countries. Its main known risk factors are associated with prolonged exposure to estrogens, are indicators of lifestyle and reproductive patterns, and therefore are difficult to modify and reducing mortality, then, it requires improving early detection and treatment strategies. Among screening procedures, which also include self-examination and clinical examination, mammography is the only technique that can offer sufficiently timely detection. In which a low energy X-rays is used to screen breast in order to assist breast detection. To ensure an accurate diagnosis, breast $\mathrm{x}$-rays images should be of high quality. In this

\footnotetext{
${ }^{1}$ https://www.who.int/news-room/fact-sheets/detail/cancer
} 
direction, multiple approaches are used for de-noising the mammogram is one approach to improve quality. In [20, 21], convolutional neural networks (CNNs) are applied to minimize the noise in mammograms. Recently, Total Variation (TV) and Non-Local Mean (NLM) algorithms are developed to mitigate some shortages of repeatable noise elimination in medical images [22].

In summary, the previous solutions in-depth related studies are still not flexible and need to be improved in terms of developing a method to remove the noise from a noisy image at different levels for getting a high-quality image depending on the selected level. Thus, this paper proposes a repeated median filtering (RMF) method that applies a median filter at a different number of iterations with different filter sizes making it more flexible for user choice.

\section{RESEARCH METHODS}

\section{A. Median Filter $(M F)$}

In image processing, before further processing, such as edge detection, it is usually necessary to first perform a certain degree of noise reduction. The filtering process using MF is a common step in image processing. It is especially useful for speckle noise and salt-and-pepper noise. Preserving the edges makes it useful in situations where edge blur is not desired.

Median filter (MF) is a non-linear digital filter technology that is often used to remove noise from images or other signals. The design idea is to check the samples in the input signal and determine whether it represents the signal. Use an observation window composed of an odd number of samples to achieve this function. The values in the observation window are sorted, and the median value in the middle of the observation window is used as the output. Then, the oldest value is discarded, new samples are obtained, and the above calculation process is repeated. The main idea of the MF is to traverse the signal entry through the entry and replace each entry with the median of the neighbor entry. The neighbor's pattern is called a "window," and it slides through the entrance to cover the entire signal. For one-dimensional signals, the most obvious windows are only the front and back items, while 2D (or higher-dimensional) signals (such as images) may have more complex window modes (such as "box" or "cross" modes). Note that if there is an odd number of entries in the window, the median is easy to define: after all entries in the window are sorted numerically, this is only the middle value. For even entries, there is more than one possible median.

Median filtering is a smoothing technique similar to linear Gaussian filtering. All smoothing techniques can effectively remove noise in smooth or smooth areas of image signal but have an adverse effect on the edges. In general, it is essential to maintain edges while reducing noise in the signal. For example, edges are critical to the visual appearance of an image. For small to medium levels of Gaussian noise, the MF is significantly better at removing noise than Gaussian blur while preserving edges for given fixed window size. However, for high noise, its performance is not better than the Gaussian blur, and it is particularly effective for speckle noise and salt and pepper noise (impulse noise). Therefore, MF is widely used in digital image processing [3].

The naive implementation described above sorts each entry in the window to find the intermediate value; however, since only the intermediate value in the list is needed, the selection algorithm can be more efficient. In addition, some types of signals (usually the case of images) are represented using integers: in these cases, the histogram is simple because updating the histogram from window to window and finding the median of the histogram is not incredibly tedious that makes it to be much more efficient. Fig. 1 illustrates an example of a median filter calculation.

\section{B. Proposed Repeated Median Filtering (RMF) Method}

The repeated median filtering (RMF) method is a nonlinear median-based processing approach that applies an $\mathrm{MF}$ on an image $N$ times to remove noises at different levels for getting a high-quality image depending on the suitable selected level. The main idea of the RMF method is very simple but more effective. In this method, the number of iterations and filter size should be defined that makes it flexible for user choice. To illustrate how the method works, Algorithm 1 describes the steps of the RMF process. In the application of the RMF method, the user needs to initialize the method's parameters, such as the number of iterations and the filter size. The large size of MF used in the approach is not suitable due to a large set of pixels that makes the MF values deviate from the values of pixels. The number of iterations makes the method is more flexible to perform the filtering process at different times. The filter size forms a $2 \mathrm{D}$ window that is a central symmetric shape that replaces the pixel at the center by the median value of pixels values inside that window.

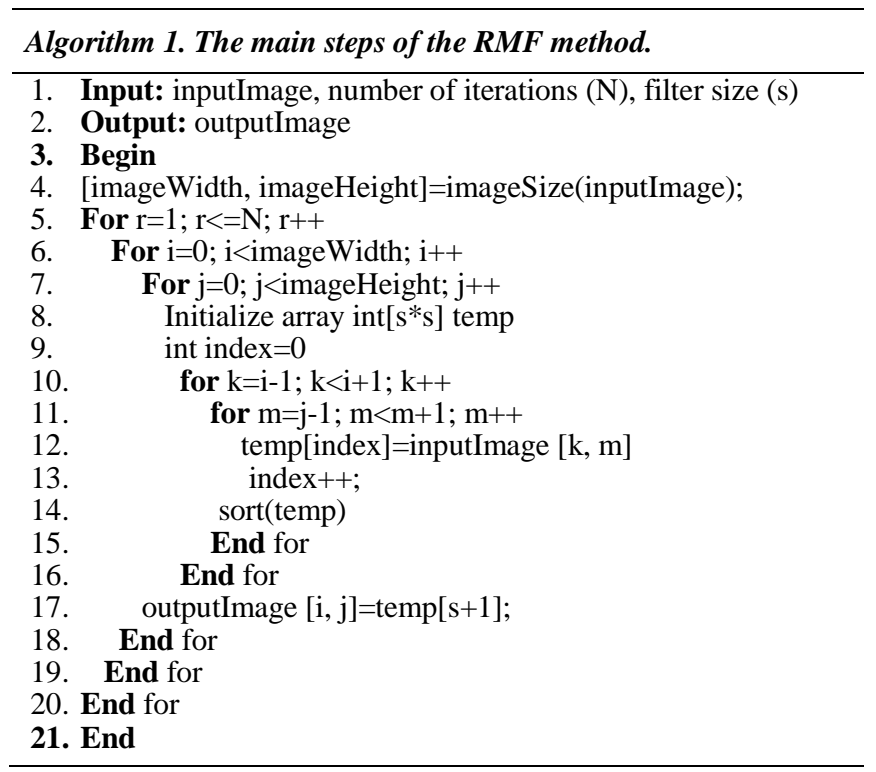




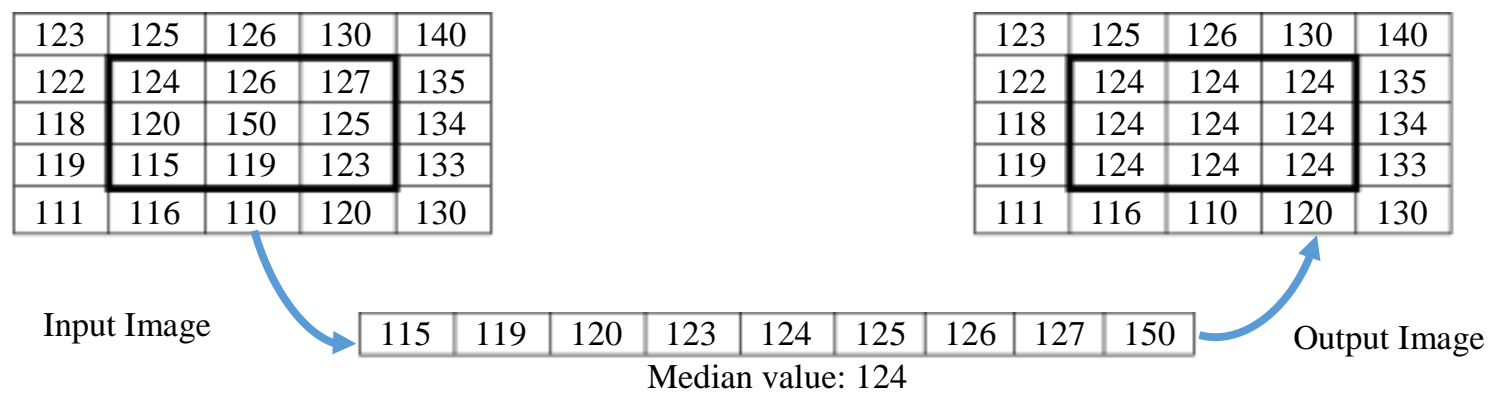

Fig. 1. An Example of a Filtering Process using MF.

\section{EXPERIMENTS AND DISCUSSION}

In this section, a set of experiments are conducted on a number of breast mammogram images taken from the database of mini- Mammogram Image Analysis Society (MIAS) to evaluate the proposed method. In addition, the results of the proposed method will be compared with the results of MF on the same images. The proposed method is implemented by using the MATLAB R2016b programming tool.

The implementation was performed on a laptop that has an Intel CPU I7 $2.2 \mathrm{GHz}$ with $16 \mathrm{~GB}$ of RAM and a Windows 10 operating system. With these experiments' configurations, the evaluation results on the test images are assessed using the peak signal-to-noise ratio (PSNR) and mean squared error (MSE) performance metrics. The experimental results and comparisons will be introduced in the following subsections.

\section{A. Dataset Mammogram Images}

The dataset mammogram images are five samples, selected from the Mini-mammogram Image Analysis Society (MIAS) database and shown in Fig. 2. All test images are in PGM format. In the experiments, these images are converted into PNG format and resized to $256 \times 256$ pixels.

\section{B. Image Quality Evaluation Measures}

The evaluation measures used to assess the proposed image denoising method are quantitative image quality measures include Mean Square Error (MSE) and Peak Signal to Noise Ratio (PSNR). These measures are computed based on original and denoised images. MSE is a cumulative value of squared errors between an original image $(\mathrm{O})$ and a denoised image (D) with 2D matrices with $m$ rows and $n$ columns. MSE has a small value if the method performs well and can be computed as [23]:

$M S E=\frac{1}{M * N} \sum_{M, N}[O(m, n)-D(m, n)]^{2}$

The second measure is the PSNR that can give a good indication of the capability of the method to remove the noises. The small value of PSNR for the denoised image means it has a poor quality [23]. PSNR can be calculated as in the following equation.

$P S N R=10 \log _{10}\left(\frac{R^{2}}{M S E}\right)$

The variable $R$ in the previous equation is the maximum fluctuation of image's pixels if the image has a data type of double floating-point, then $R$ is one, and if the image has a data type of 8-bit unsigned integer, then $R$ is 255 .

\section{Results and Discussion}

To validate the proposed denoising method, all evaluation images are degraded artificially using speckle noise with a different ratio of $0.5 \%$ and $1 \%$. Besides, they are also degraded using salt and pepper noise with a different ratio of $10 \%$ and $20 \%$, respectively. The experimental results of the proposed filtering method on noised images are assessed based on the adopted image quality evaluation measures. During the experiments, the number of iterations and filter size are initialized. Initializing the number of iterations is critical to determine the quality of denoised image. Therefore, a set of experiments are conducted to select the best value of the number of iterations. Tables I and II list the quantitative results of MSE and PSNR measures under a different number of iterations $(\mathrm{N})$.

From Tables I and II, the best quantitative results of MSE and PSNR are when the value of $\mathrm{N}$ is 2 . Thus, this value is selected for the method to remove the images' noise. Tables III and IV exhibits the experimental results of MSE and PSNR for the proposed method at different noise levels of test images degraded with speckle noise.

TABLE I. QUANTITATIVE RESULTS OF MSE AND PSNR MEASURES OF
RMF METHOD UNDER A DIFFERENT NUMBER OF ITERATIONS (N) FOR SPECKLE NOISE WITH A $1 \%$ NOISE RATIO ON MDB015.PGM IMAGE

\begin{tabular}{|l|l|l|}
\hline $\mathbf{N}$ & MSE & PSNR \\
\hline 1 & 0.00036 & 82.5685 \\
\hline 2 & 0.00030 & 83.3691 \\
\hline 3 & 0.00032 & 83.2037 \\
\hline 4 & 0.00034 & 82.9138 \\
\hline 5 & 0.00036 & 82.5974 \\
\hline
\end{tabular}

TABLE II. QUANTITATIVE RESULTS OF MSE AND PSNR MEASURES OF RMF METHOD UNDER A DIFFERENT NUMBER OF ITERATIONS (N) FOR SALT AND PEPPER NOISE WITH A 20\% NOISE RATIO ON MDB015.PGM IMAGE

\begin{tabular}{|l|l|l|}
\hline $\mathbf{N}$ & MSE & PSNR \\
\hline 1 & 0.00110 & 77.6215 \\
\hline 2 & 0.00035 & 82.7175 \\
\hline 3 & 0.00037 & 82.5298 \\
\hline 4 & 0.00040 & 82.1220 \\
\hline 5 & 0.00044 & 81.7712 \\
\hline
\end{tabular}


As shown in Tables III and IV, the proposed method achieves high values of PSNR and low values of MSE. These results validate the effectiveness of the method to remove the speckle noises from the test images. Fig. 3 visualizes an example of noised and denoised images.

Fig. 2 shows how the noisy images are improved by using the RMF method that removes the speckle noise that has a ratio of $1 \%$. Tables $\mathrm{V}$ and $\mathrm{VI}$ demonstrate the quantitative results of MSE and PSNR for the proposed method on the test images that are degraded with salt and pepper noise at different noise levels of $10 \%$ and $20 \%$. Fig. 4 visualizes an example of noised and denoised images.

To compare the proposed RMF method with the MF method, Tables $\mathrm{V}$ and VI show the results of PSNR of denoised images using RMF and MF methods. Table V displays the results of PSNR on the test images that are degraded with speckle-noise at $1 \%$ of the noise level. Similarly, Table VI exhibits the results of PSNR on the test images that are degraded with salt and pepper noise at $20 \%$ of the noise level.
TABLE III. QUANTITATIVE RESULTS OF MSE AND PSNR MEASURES OF RMF METHOD FOR SPECKLE NOISE WITH A $0.5 \%$ NOISE RATIO ON TEST IMAGES

\begin{tabular}{|l|l|l|}
\hline Image No. & MSE & PSNR \\
\hline mdb005.pgm & 0.00030 & 83.4473 \\
\hline mdb010.pgm & 0.00014 & 86.6788 \\
\hline mdb012.pgm & 0.00038 & 82.3583 \\
\hline mdb013.pgm & 0.00025 & 84.2229 \\
\hline mdb015.pgm & 0.00021 & 84.8947 \\
\hline
\end{tabular}

TABLE IV. QUANTITATIVE RESUlTS OF MSE AND PSNR MEASURES OF RMF METHOD FOR SPECKLE NOISE WITH A $1 \%$ NOISE RATIO ON TEST IMAGES

\begin{tabular}{|l|l|l|}
\hline Image No. & MSE & PSNR \\
\hline mdb005.pgm & 0.00042 & 81.9013 \\
\hline mdb010.pgm & 0.00023 & 84.4980 \\
\hline mdb012.pgm & 0.00055 & 80.7610 \\
\hline mdb013.pgm & 0.00036 & 82.5686 \\
\hline mdb015.pgm & 0.00030 & 83.3691 \\
\hline
\end{tabular}
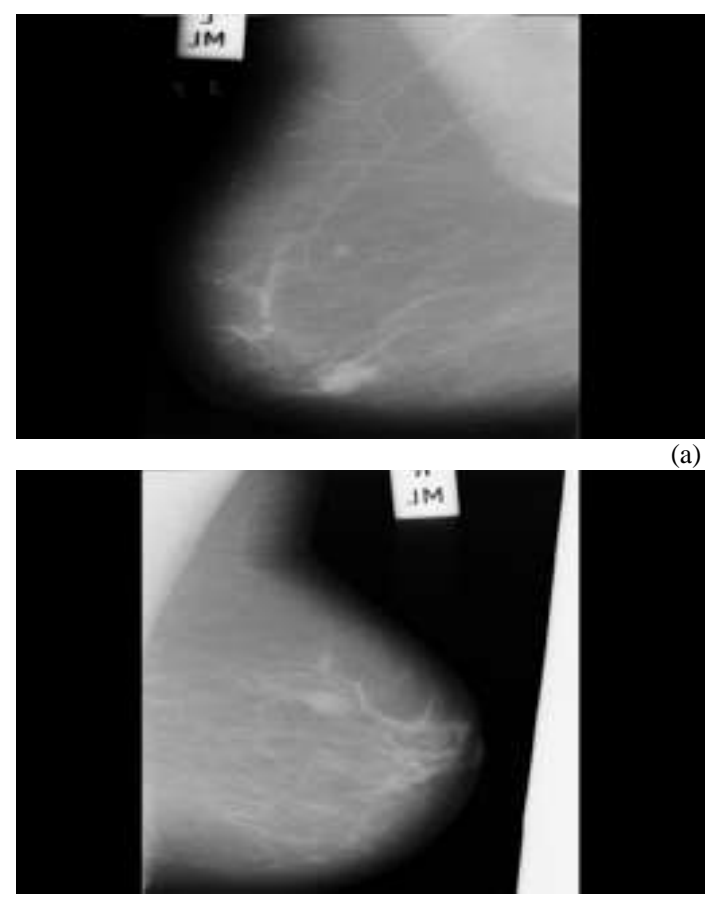

(a)

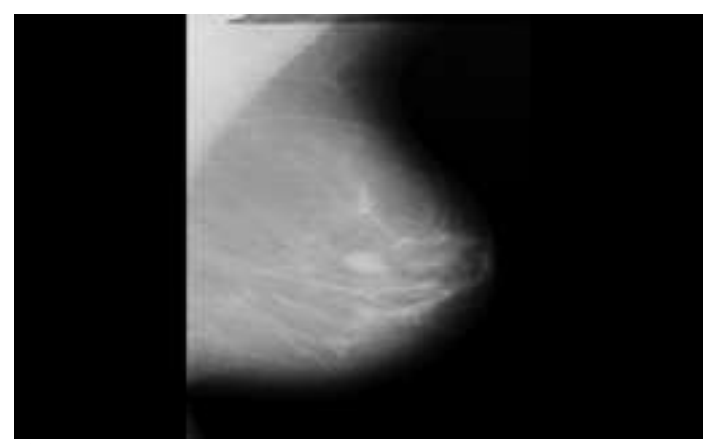

(b)

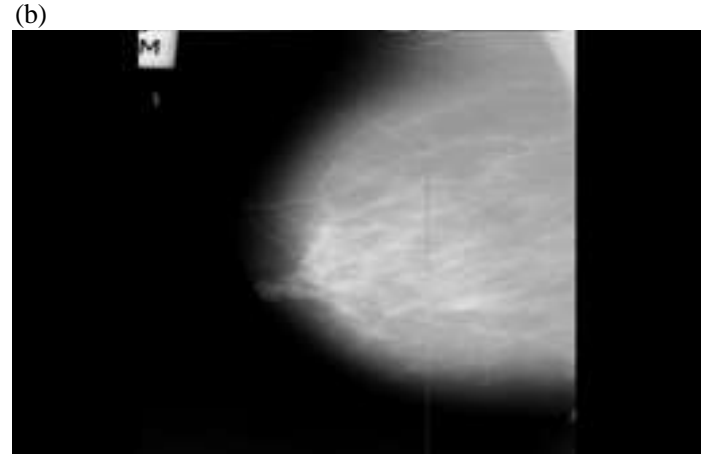

(d)

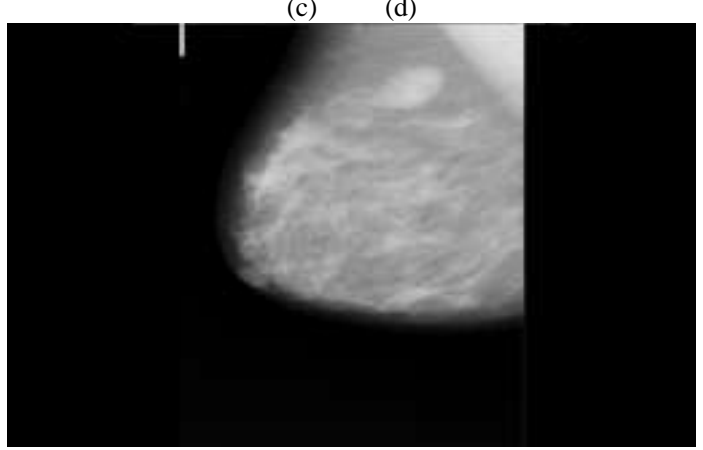

(e)

Fig. 2. Test Evaluation Images Taken from Mini- Mammogram Image Analysis Society (MIAS) Database under Images Names: (a) mdb005.pgm, (b) mdb010.pgm, (c) mdb012.pgm, (d) mdb013.pgm, and (e) mdb015.pgm. 


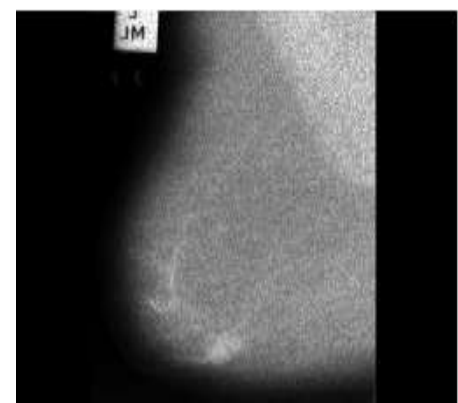

(a)

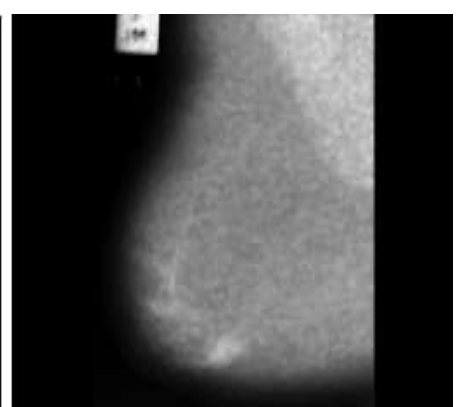

(b)

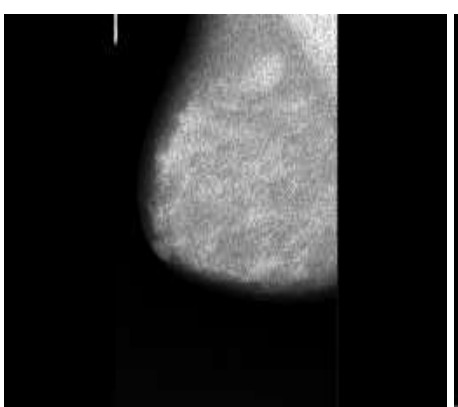

(c)

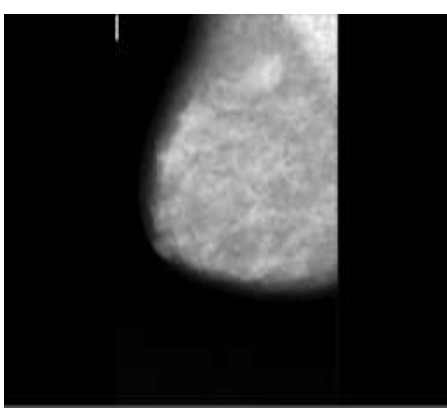

(d)

Fig. 3. A Visualization Example of Noised and Denoised using the RMF Method for Speckle Noise Removal with 1\% Noise ratio: (a) and (c) Noisy Images, (b) and (d) Denoised Images.

TABLE V. COMPARISON RESULTS OF PSNR MEASURE FOR RMF AND MF METHODS FOR SPECKLE NOISE WITH A 1\% NOISE RATIO ON TEST IMAGES

\begin{tabular}{|l|l|l|}
\hline Image No. & RMF PSNR & MF PSNR \\
\hline mdb005.pgm & 81.9013 & 80.9391 \\
\hline mdb010.pgm & 84.4980 & 83.0336 \\
\hline mdb012.pgm & 80.7610 & 80.0645 \\
\hline mdb013.pgm & 82.5686 & 81.4102 \\
\hline mdb015.pgm & 83.3691 & 82.5685 \\
\hline
\end{tabular}

TABLE VI. COMPARISON RESULTS OF PSNR MEASURE FOR RMF AND MF METHODS FOR SALT AND PEPPER NOISE WITH 20\% RATIO ON TEST IMAGES

\begin{tabular}{|l|l|l|}
\hline Image No. & RMF PSNR & MF PSNR \\
\hline mdb005.pgm & 81.8447 & 77.6831 \\
\hline mdb010.pgm & 84.6203 & 77.8431 \\
\hline mdb012.pgm & 77.0829 & 74.7745 \\
\hline mdb013.pgm & 83.0218 & 77.8119 \\
\hline mdb015.pgm & 82.7175 & 77.6215 \\
\hline
\end{tabular}

Besides, Fig. 5 and 6 visualize the results of MSE for the RMF and MF methods applied on the test images that are corrupted by speckle noise with $1 \%$ noise ratio and salt and pepper noise with $20 \%$ noise ratio.

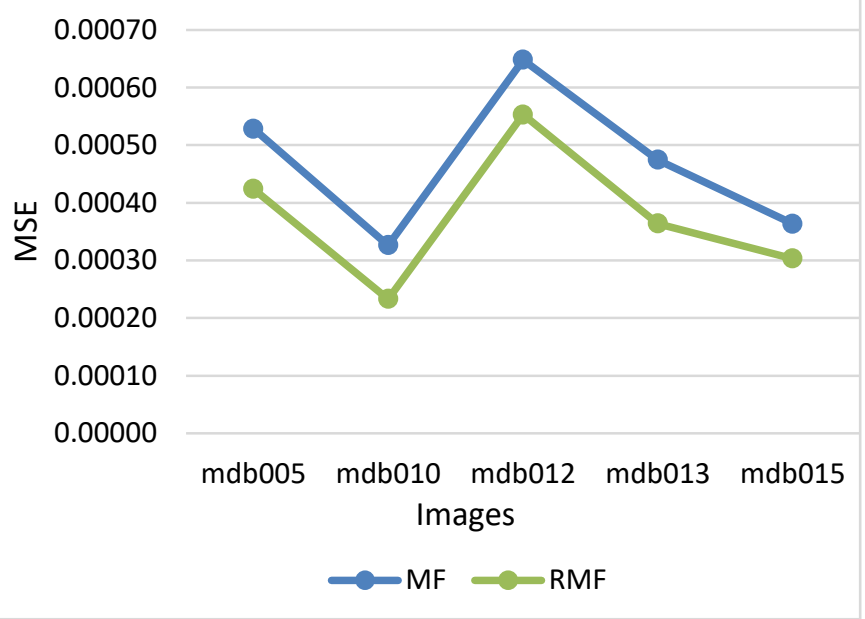

Fig. 4. A Visualization of MSE Results for the RMF and MF Methods on the Test Images Corrupted by Speckle Noise with a 1\% Noise Ratio.

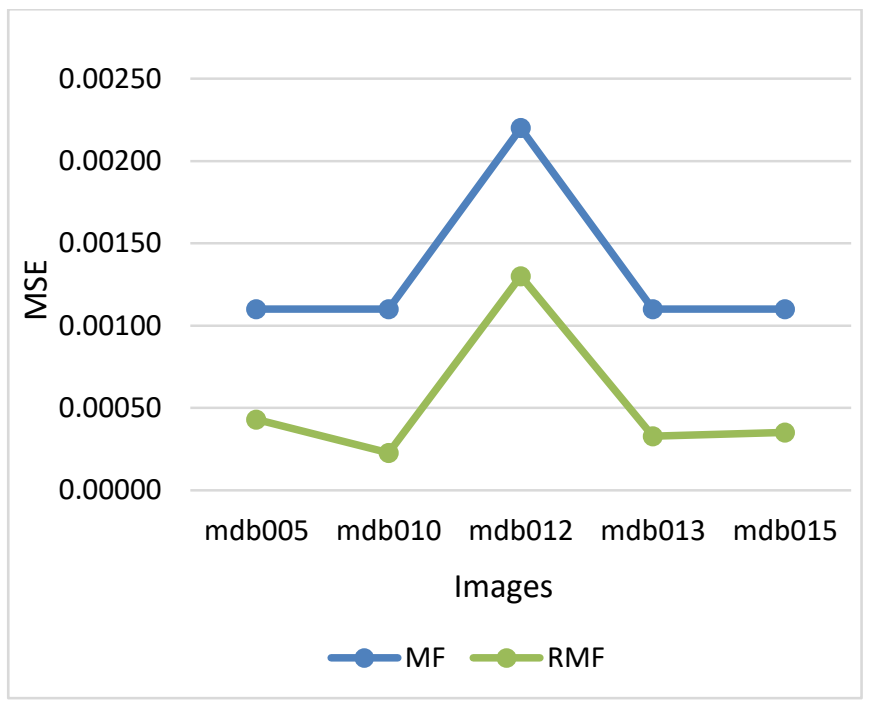

Fig. 5. A Visualization of MSE Results for the RMF and MF Methods on the Test Images Corrupted by Salt and Pepper Noise with a 20\% Noise Ratio.

From comparison results in Tables V and VI, as well as Fig. 4 and 5, it is clear that the proposed RMF method outperforms the MF method in terms of PSNR for all test images. For the speckle noise, an improvement of the denoised images is greater than 1\%. Furthermore, for the salt and pepper noise, the proposed method achieves a significant high PSNR result.

\section{CONCLUSIONS AND FUTURE WORK}

In the medical field, mammogram analysis is one of the most important procedures for breast cancer detection and early diagnosis. During the image acquisition process of mammograms, these images may be contained some noises due to the change of illumination and sensor error. Hence, it is necessary to remove these noises without affecting the edges and fine details, achieving an effective diagnosis of beast images. Therefore, in this paper, a repeated median filtering (RMF) method is proposed for denoising mammogram images. This method is able to enhance the digital mammogram images in a special domain to preserve the useful information of images. To evaluate the proposed method, a number of experiments are conducted on a dataset of different mammogram images to evaluate the proposed method using a set of image quality metrics. Experimental results are reported by computing the image quality metrics 
between the original clean images and denoised images that are corrupted by different levels of simulated speckle noise as well as salt and paper noise. Evaluation quality metrics showed that the repeated median filter method achieves a higher result than the related traditional median filter method.

\section{ACKNOWLEDGMENTS}

The author is thankful to the Deanship of Scientific Research, College of Computer and Information Sciences (CCIS) at King Saud University for funding this research.

\section{REFERENCES}

[1] E.-L. Chen, P.-C. Chung, C.-L. Chen, H.-M. Tsai, and C.-I. Chang, "An automatic diagnostic system for CT liver image classification," IEEE transactions on biomedical engineering, vol. 45, no. 6, pp. 783-794, 1998.

[2] W. Cao, X. An, L. Cong, C. Lyu, Q. Zhou, and R. Guo, "Application of Deep Learning in Quantitative Analysis of 2 - Dimensional Ultrasound Imaging of Nonalcoholic Fatty Liver Disease," Journal of Ultrasound in Medicine, vol. 39, no. 1, pp. 51-59, 2020.

[3] A. Gumaei, M. M. Hassan, M. R. Hassan, A. Alelaiwi, and G. Fortino, "A hybrid feature extraction method with regularized extreme learning machine for brain tumor classification," IEEE Access, vol. 7, pp. 3626636273, 2019.

[4] J. Amin, M. Sharif, N. Gul, M. Yasmin, and S. A. Shad, "Brain tumor classification based on DWT fusion of MRI sequences using convolutional neural network," Pattern Recognition Letters, vol. 129, pp. 115-122, 2020.

[5] H. AlSalman and N. Almutairi, "IDSS: An Intelligent Decision Support System for Breast Cancer Diagnosis," in 2019 2nd International Conference on Computer Applications \& Information Security (ICCAIS), 2019, pp. 1-6: IEEE.

[6] A. Gumaei, A. El-Zaart, M. Hussien, and M. Berbar, "Breast segmentation using k-means algorithm with a mixture of gamma distributions," in 2012 Symposium on Broadband Networks and Fast Internet (RELABIRA), 2012, pp. 97-102: IEEE.

[7] H. AlSalman, "Enhancing Digital Mammogram Images using Bandpass Filters in Frequency Domain," International Journal of Computer Science and Network Security, vol. 19, no. 11, pp. 107-113.

[8] L. Fan, F. Zhang, H. Fan, and C. Zhang, "Brief review of image denoising techniques," Visual Computing for Industry, Biomedicine, Art, vol. 2, no. 1, p. 7, 2019.

[9] M. C. Motwani, M. C. Gadiya, R. C. Motwani, and F. C. Harris, "Survey of image denoising techniques," in Proceedings of GSPX, 2004, pp. 2730 .
[10] P. Jain and V. Tyagi, "A survey of edge-preserving image denoising methods," Information Systems Frontiers, vol. 18, no. 1, pp. 159-170, 2016.

[11] M. Diwakar and M. Kumar, "A review on CT image noise and its denoising," Biomedical Signal Processing Control, vol. 42, pp. 73-88, 2018.

[12] P. Milanfar, "A tour of modern image filtering: New insights and methods, both practical and theoretical," IEEE signal processing magazine, vol. 30, no. 1, pp. 106-128, 2012.

[13] R. C. Gonzalez, R. E. Woods, and S. L. Eddins, Digital image processing using MATLAB (Pearson Education India). 2004.

[14] H. Ibrahim, N. S. P. Kong, and T. F. Ng, "Simple adaptive median filter for the removal of impulse noise from highly corrupted images," IEEE Transactions on Consumer Electronics, vol. 54, no. 4, pp. 1920-1927, 2008.

[15] S. Agarwal and S. Chand, "Median Filtering Detection Using Markov Process in Digital Images," in Advances in Biomedical Engineering and Technology: Springer, pp. 35-42.

[16] S. Ghael, A. M. Sayeed, and R. G. Baraniuk, "Improved wavelet denoising via empirical Wiener filtering," in SPIE Technical Conference on Wavelet Applications in Signal Processing, 1997.

[17] J. Mehena, "Medical Image Edge Detection Using Modified Morphological Edge Detection Approach," 2019.

[18] Y. Yang, D. S. Park, S. Huang, and N. Rao, "Medical image fusion via an effective wavelet-based approach," EURASIP Journal on Advances in Signal Processing, vol. 2010, pp. 1-13, 2010.

[19] J.-L. Starck, E. J. Candès, and D. L. Donoho, "The curvelet transform for image denoising," IEEE Transactions on image processing, vol. 11, no. 6, pp. 670-684, 2002.

[20] D. Abdelhafiz, C. Yang, R. Ammar, and S. Nabavi, "Deep convolutional neural networks for mammography: advances, challenges and applications," BMC bioinformatics, vol. 20, no. 11, p. 281, 2019.

[21] L. Li, Y. Chen, Z. Shen, X. Zhang, J. Sang, Y. Ding, X. Yang, J. Li, M. Chen, and C. Jin, "Convolutional neural network for the diagnosis of early gastric cancer based on magnifying narrow band imaging," Gastric Cancer, pp. 1-7, 2019.

[22] S. Lee, S. J. Park, J. M. Jeon, M.-H. Lee, D. Y. Ryu, E. Lee, S.-H. Kang, and Y. Lee, "Noise removal in medical mammography images using fast non-local means denoising algorithm for early breast cancer detection: a phantom study," Optik, vol. 180, pp. 569-575, 2019.

[23] R. Sammouda, A. M. S. Al-Salman, A. Gumaei, and N. Tagoug, "An efficient image denoising method for wireless multimedia sensor networks based on DT-CWT," International Journal of Distributed Sensor Networks, vol. 11, no. 11, p. 632568, 2015. 